\title{
Excitons and trions in spherical semiconductor quantum dots
}

\author{
I.M. Kupchak, Yu.V. Kryuchenko, D.V. Korbutyak \\ V. Lashkaryov Institute of Semiconductor Physics, NAS of Ukraine, \\ 45, prospect Nauky, 03028 Kyiv, Ukraine \\ Phone: +38(044) 525 6391, e-mail:div47@isp.kiev.ua
}

\begin{abstract}
An original iterative procedure has been developed to obtain energy spectrum of neutral and charged excitons (positive and negative trions) in spherical semiconductor quantum dots (QD) imbedded into a dielectric material. Numerical calculations are made using the effective mass approximation and Hartree-Fock method. A combined effect of heterointerface polarization (image force potential) and finite band-off-sets on the energy spectrum of excitons and trions in QDs is considered for the first time. It is shown that binding energies of excitons and trions in such QDs can be substantially larger than those in bulk semiconductors due to spatial and "dielectric" confinement effects.
\end{abstract}

Keywords: semiconductor quantum dot, quantum size effect, image force potential, excitons, trions, energy spectrum, binding energy.

Manuscript received 30.11.05; accepted for publication 15.12.05.

\section{Introduction}

The neutral or charged excitons (trions) are quasiparticles composed of two or three interacting charge carriers, e.g. of an electron and a hole (neutral exciton), of two electrons and a hole (a negative trion $X^{-}$) or of two holes and one electron (a positive trion $X^{+}$). In semiconductors, the existence of three-particle complexes bound by the Coulomb interaction was predicted in 1958 by Lampert [1]. As was shown in that work, in bulk semiconductors these charged electronhole states are energetically favourable as compared to a three-particle state "neutral exciton plus free charge carrier". However, their binding energy turned out to be very small (usually, less than $1 \mathrm{meV}$ ) and for this reason the experimental investigation of these states in bulk semiconductors is substantially complicated. Nevertheless, the trions were observed for example in bulk silicon [2] and germanium [3]. Very interesting is the question about exciton and trion states in semiconductor systems with spatial confinement when partial or total quantization of electron and/or hole energy spectra occurs. As was shown in a number of experimental and theoretical works on quantum wells and quantum wires (e.g., see [4-19], binding energies of exciton and trion states increase substantially in such structures. For example, in accordance with theoretical calculations in the case of semiconductor quantum wells [20] the trion binding energy should be an order of magnitude greater than that in bulk semiconductors. In reality, this fact has been confirmed experimentally [21]. Spatially confined trions were observed in the structures with QDs as well. Of special interest are the investigations of photoluminescence spectra of single QDs $[22,23]$ where lines caused by trion transitions are clearly revealed.

In addition to spatial confinement in many semiconductor nanostructures the effect of dielectric confinement due to large dielectric mismatch with environment plays very important role, too [see, e.g. 2427]. Unfortunately, in a majority of previous works this effect has been considered theoretically only in the approximation of infinitely high barriers, when wave functions of electron and hole confined states turn to zero at the interfaces. It is clear that with a decrease of characteristic size of nanocrystals such approximation becomes too bad for the finite band-off-sets in real semiconductor-dielectric nanostructures.

In this work, we consider theoretically the exciton and trion states that can be formed and radiate in semiconductor QDs located in a dielectric matrix. Both finite band-off-sets and dielectric confinement effects are taken into account as in our previous works [9, 28] on quantum wells and quantum wires. We exploit here a simplified solid state model based on the effective mass approximation. Moreover, only heavy-hole subband forming the lowest in energy confined hole states in QDs has been taken into account in our calculations. Additional effects which can arise due to subband mixing, anisotropy, magnetic and electric fields, etc. will be considered in future. 
If an electron and a hole move simultaneously in QD, then the energy of the ground radiative electron-hole (exciton) transition in such a QD can be written as follows:

$$
E_{X}=E_{g}+E_{e}+E_{h}-S_{X}
$$

where $E_{g}$ is the bandgap width of bulk material containing QDs, $E_{e}$ and $E_{h}$ are the lowest single-particle confinement energies in these QDs, respectively, $S_{X}$ is the exciton binding energy caused by the Coulomb interaction between an electron and a hole in QDs.

If three carriers (two electrons and a hole or two holes and an electron) move simultaneously in a QD, then the energy of the ground radiative electron-hole transition in such a system may be written as

$E_{X^{-}}=E_{X}-S_{X^{-}}$

for a negative trion case and

$E_{X^{+}}=E_{X}-S_{X^{+}}$

for a positive trion case. In Eqs (2) and (3) the values $-S_{X^{-}}$and $-S_{X^{+}}$are the energy shifts of the ground radiative trion transitions with respect to the energy of the ground exciton transition. If these values are negative, the corresponding trion states are energetically favourable as compared to the states of a three-particle system in the form of "free" exciton and "free" charge carrier. In other words, the values $-S_{X^{-}}$and $-S_{X^{+}}$are the trion "binding" energy with respect to the virtual state of non-interacting exciton and charge carrier.

\section{Model and results}

The trion states, where the pair Coulomb interaction between all the quasi-particles of which the trion is composed plays a substantial role, in a first approximation may be built of single-particle states of a model Hamiltonian for a spherically-symmetric semiconductor QD in a dielectric matrix. In this case, the conduction and valence band-off-sets at the "semiconductor QD dielectric matrix" heterointerface form rectangular potential energy gaps both for electrons and holes inside the QD region. The corresponding coordinate dependences of the electron and hole potential energies $U_{c}$ and $U_{v}$ caused by the band-off-sets can be written as

$U_{c(v)}(r)=\left\{\begin{array}{ll}0, & \text { if } r<R \\ U_{e(h)}, & \text { if } r>R\end{array}\right.$,

where $R$ is the QD radius, $U_{e(h)}$ are the band-off-set values.

In the case when the QD material dielectric constant $\varepsilon_{1}$ differs from that of the ambient $\varepsilon_{2}$, the polarization of the heterointerface by a charged point particle forms an additional image forces potential field. The potential energy of a particle in this field (self-action energy) can be expressed using the Green function $G\left(\mathbf{x}, \mathbf{x}^{\prime}\right)$ of the following Poisson equation for the point positive unit charge:

$\Delta_{x} G\left(\mathbf{x}, \mathbf{x}^{\prime}\right)=-\frac{4 \pi}{\varepsilon} \delta\left(\mathbf{x}-\mathbf{x}^{\prime}\right)$,

where $\varepsilon=\varepsilon_{1}$ if $r<R$ and $\varepsilon=\varepsilon_{2}$ if $r>R, \mathbf{x}^{\prime}$ is the coordinate of a point unit charge that forms the electrostatic potential $G\left(\mathbf{x}, \mathbf{x}^{\prime}\right)$ at the point $\mathbf{x}$. The solution of this equation can be conveniently written in a form of the following expansion in spherical harmonics $Y_{l m}[29]$ :

$G\left(\mathbf{x}, \mathbf{x}^{\prime}\right)=\sum_{l=0}^{\infty} \sum_{m=-l}^{l} g_{l} Y_{l m}^{*}(\Omega) Y_{l m}\left(\Omega^{\prime}\right)$.

In the case, when both the charge and the observation point are located inside the QD, i.e., when both $r^{\prime}<R$ and $r<R$ then

$$
\begin{aligned}
& g_{l}\left(r, r^{\prime}\right)=\frac{4 \pi}{\varepsilon_{1}(2 l+1)} \frac{r_{<}^{l}}{r_{>}^{l+1}}+ \\
& +\frac{4 \pi}{\varepsilon_{1}(2 l+1)} \frac{\left(\varepsilon_{1}-\varepsilon_{2}\right)(l+1)}{\varepsilon_{1} l+\varepsilon_{2}(l+1)} \frac{\left(r_{>} r_{<}\right)^{l}}{R^{2 l+1}},
\end{aligned}
$$

where the radial coordinate $r_{>}$is the greatest of the $r$ and $r^{\prime}$ coordinates, while $r_{<}$is the least one. In the case when both the charge and the observation point are located beyond the QD in the barrier region, i.e., when both $r^{\prime}>R$ and $r>R$, the corresponding expression has the form of

$$
\begin{aligned}
& g_{l}\left(r, r^{\prime}\right)=\frac{4 \pi}{\varepsilon_{2}(2 l+1)} \frac{r_{<}^{l}}{r_{>}^{l+1}}+ \\
& +\frac{4 \pi}{\varepsilon_{2}(2 l+1)} \frac{l\left(\varepsilon_{2}-\varepsilon_{1}\right)}{\varepsilon_{1} l+\varepsilon_{2}(l+1)} \frac{R^{2 l+1}}{\left(r_{>} r_{<}\right)^{l+1}} .
\end{aligned}
$$

Finally, when the charge and the observation point are at different sides of the heterointerface

$$
\begin{aligned}
& g_{l}\left(r, r^{\prime}\right)=\frac{4 \pi}{\bar{\varepsilon}(2 l+1)} \frac{r_{<}^{l}}{r_{>}^{l+1}}+ \\
& +\frac{4 \pi}{(2 l+1)} \frac{\left(\varepsilon_{1}-\varepsilon_{2}\right)}{\left(\varepsilon_{1}+\varepsilon_{2}\right)} \frac{1}{\varepsilon_{1} l+\varepsilon_{2}(l+1)} \frac{r_{<}^{l}}{r_{>}^{l+1}},
\end{aligned}
$$

where $\bar{\varepsilon}=\left(\varepsilon_{1}+\varepsilon_{2}\right) / 2$. Taking into account the wellknown expression

$\frac{1}{\left|\mathbf{x}-\mathbf{x}^{\prime}\right|}=4 \pi \sum_{l=0}^{\infty} \sum_{m=-l}^{l} \frac{1}{2 l+1} \frac{r_{<}^{l}}{r_{>}^{l+1}} Y_{l m}^{*}\left(\theta^{\prime}, \varphi^{\prime}\right) Y_{l m}(\theta, \varphi)$,

it is clear that the first term in the right-hand sides of Eqs (7) - (9) corresponds to the usual Coulomb field of a point positive unit charge in the medium with the effective dielectric constant $\varepsilon_{1}, \varepsilon_{2}$, and $\bar{\varepsilon}=\left(\varepsilon_{1}+\varepsilon_{2}\right) / 2$, 
respectively. The second term in the right-hand sides of Eqs (7)-(9) is the image forces field component that arises due to the QD heterointerface polarization by the point charge.

The self-action potential energy of the charge $e$ located at the point $\mathbf{x}$ is equal to $U_{S}(r)=e^{2} G^{\prime}(\mathbf{x}, \mathbf{x}) / 2$, where the prime at the Green function indicates only the part of this function that is associated with the second term in Eqs (7) and (8), i.e. with the self-image field. Thus, the potential energy of a charged particle in the image force field induced by this particle itself (the selfaction potential energy) can be expressed by the following formulas:

$U_{s}(r)=\frac{e^{2}}{2 R} \frac{\varepsilon_{1}-\varepsilon_{2}}{\varepsilon_{1}} \sum_{l=0}^{\infty} \frac{l+1}{\varepsilon_{1} l+\varepsilon_{2}(l+1)}\left(\frac{r}{R}\right)^{2 l}, \quad$ if $r<R$

and

$U_{s}(r)=-\frac{e^{2}}{2 R} \frac{\varepsilon_{1}-\varepsilon_{2}}{\varepsilon_{2}} \sum_{l=0}^{\infty} \frac{l}{\varepsilon_{1} l+\varepsilon_{2}(l+1)}\left(\frac{R}{r}\right)^{2(l+1)}$, if $r>R$.

The classical point charge electrostatic potential and, hence, the self-action potential energy has a nonphysical diverging behavior near the heterointerface $U_{s}(r) \sim e^{2}\left(\varepsilon_{1}-\varepsilon_{2}\right) /\left(4 \varepsilon_{1,2} z\left(\varepsilon_{1}+\varepsilon_{2}\right)\right)$, where $z=|R-r|$ is the distance of the charged particle from the heterointerface. However, in real systems a transient layer between the QD and the barrier region always exists, therefore, the electrostatic potential is in fact continuous [30]. The continuity of the electrostatic potential at the heterointerface and in the transient layer also follows from the spatial dispersion account (i.e. account of a nonlocal character of interaction between the charge and the induced field). Rigorous calculations of the self-action potential energy in the transient layer is a rather difficult problem in this case. However, if we are interested only in one-particle self-energy shifts, then it is not so necessary to know the exact solution of this difficult problem in the transient region due to the fact that contributions to self-energy shifts from the internal part of the thin transient layer (i.e., that belonging to the QD region) and external part (belonging to the barrier region) will practically compensate each other and will give, as a result, only a small correction to the total single-particle energy. Therefore, to a first approximation, the exact total single-particle potential energies $U_{c(v)}+U_{s}$ within the thin transient layer (of the order of interatomic distance) around the heterointerface can be simply approximated by a polynomial over the distance $z=(R-r)$. In particular, we used in our calculations a cubic polynomial.

For example, Fig. 1 illustrates the energy diagram for electrons and holes in the case of a $\mathrm{Si}_{-} \mathrm{SiO}_{2} \mathrm{QD}$ of $1.5 \mathrm{~nm}$ in diameter. Thin solid lines correspond to the ordinary potential energy behaviour in the absence of the image forces field, while thick solid lines show the potential energy gaps, when self-action is taken into account. The self-action potential energy shift $U_{s}(r)$ at the center of the QD takes the value

$U_{s}(0)=\frac{e^{2}}{2 R} \frac{\varepsilon_{1}-\varepsilon_{2}}{\varepsilon_{1} \varepsilon_{2}}$.

The smaller is the QD, the greater is the shift of the potential gap bottom caused by the QD heterointerface polarization. Therefore, by finding the energy levels of single-particle states in a semiconductor QD within a dielectric matrix, it makes sense to account for this shift already from the very beginning, i.e., calculate electron (hole) confinement energies in rectangular spherical potential gaps of the depth $\tilde{U}_{e(h)}=U_{e(h)}-U_{s}(0)$ rather than $U_{e(h)}$. Respectively, in the final expression for the total energy of the electron-hole transition, these shifts of the bottoms of the potential gaps for an electron and a hole can be easily taken into account by adding simply $2 U_{s}(0)$. Thus, the total single-particle potential energies in the Schroedinger equation can be written in the form of $\tilde{U}_{c(v)}(r)+\tilde{U}_{s}(r)$, where $\tilde{U}_{c(v)}(r)=0$ for $r<R$ and $\tilde{U}_{c(v)}(r)=\tilde{U}_{e(h)}$ for $r>R$, while $\tilde{U}_{s}(r)=U_{s}(r)$ for $r>R$ and $\tilde{U}_{s}(r)=U_{s}(r)-U_{s}(0)$ for $r<R$. With respect to the bottoms of potential gaps changed by selfaction, the energy spectrum of single-particle states $\tilde{E}_{i, \alpha}$ is determined by the single-particle Schroedinger equations $\hat{H}_{i} \Psi_{\alpha}^{i}\left(\mathbf{r}_{i}\right)=\tilde{E}_{i, \alpha} \Psi_{\alpha}^{i}\left(\mathbf{r}_{i}\right)$, where

$$
\hat{H}_{i}=-\frac{\hbar^{2}}{2 m_{i}\left(r_{i}\right)} \Delta_{i}+\tilde{U}_{c(v)}\left(r_{i}\right)+\tilde{U}_{s}\left(r_{i}\right)
$$

Here, the index $i$ serves to denote an electron $(e)$ or a hole $(h), m_{i}\left(r_{i}\right)$ is the effective mass that takes the values $m_{i 1}$ in the QD region and $m_{i 2}$ in the barrier region. The wave functions $\Psi_{\alpha}^{i}$ of single-particle states localized in

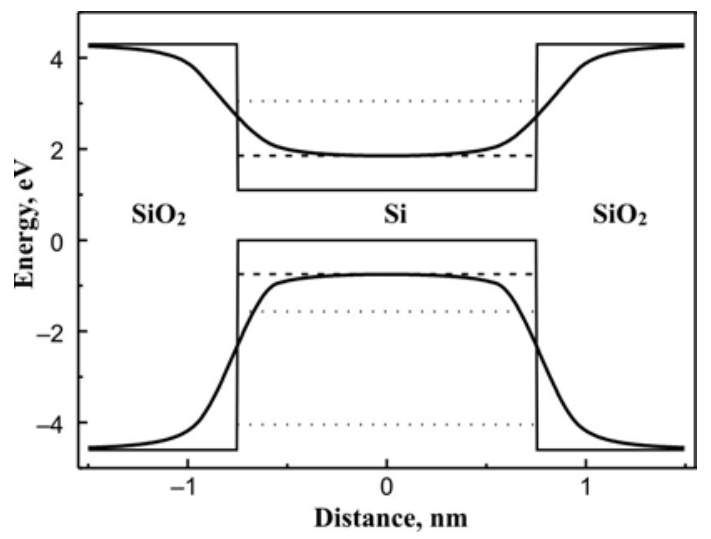

Fig. 1. Schematic potential energy diagram for electrons and holes in the case of $\mathrm{Si}_{-} \mathrm{SiO}_{2}$ quantum dot of $1.5 \mathrm{~nm}$ in diameter. Dotted lines show the energies of confined electron and hole $s$-states in the rectangular sphericallysymmetrical potential gaps with the bottoms shown by dashed lines. 
such potential gaps can be found in the form of an expansion in wave functions $\varphi_{i, k}\left(r_{i}\right)$ of a model Hamiltonian describing a confined movement of the particles in rectangular spherically-symmetrical potential gaps $\tilde{U}_{c(v)}(r)$ (the bottoms of these gaps are shown in Fig. 1 by dashed lines):

$\Psi_{\alpha}^{i}\left(\mathbf{r}_{i}\right)=\sum_{k} C_{i, k}^{\alpha} \varphi_{i, k}\left(\mathbf{r}_{i}\right)$.

To abbreviate the notation, the index $k$ is used here to denote the standard set of quantum numbers $\{n, l, m\}$ for a spherically-symmetrical system, the quantum numbers $\alpha=0,1,2 \ldots$ characterize energy levels of single-particle states in a QD when self-action potential energy (third term in the right-hand side of expression (14)) is taken into account, too.

The wave functions $\varphi_{i, k}\left(\mathbf{r}_{i}\right)$ have the following form:

$$
\begin{aligned}
& \varphi_{i, n l m}\left(\mathbf{r}_{i}\right)=N_{i, n l}\left[\theta\left(R-r_{i}\right) j_{l}\left(k_{i, n l} r_{i}\right)+\right. \\
& \left.+\theta\left(r_{i}-R\right) \frac{j_{l}\left(k_{i, n l} R\right)}{k_{l}\left(\eta_{i, n l} R\right)} k_{l}\left(\eta_{i, n l} r_{i}\right)\right] Y_{l m}\left(\Omega_{i}\right),
\end{aligned}
$$

where $\theta(x)$ is a step-function, $N_{i, n l}$ is the normalization coefficient, $k_{i, n l}=\left[2 m_{i 1} E_{i, n l}\right]^{1 / 2} / \hbar, \eta_{i, n l}=\left[2 m_{i 2}\left(\tilde{U}_{i}-\right.\right.$ $\left.\left.-E_{i, n l}\right)\right]^{1 / 2} / \hbar, j_{l}$ and $k_{l}$ are the usual and modified spherical Bessel functions. The corresponding singleparticle confinement energies $E_{i, n l}$ in such rectangular spherically-symmetrical potential gaps can be found from the equation

$\frac{k_{i} R}{m_{i 1}} \frac{j_{l+1}\left(k_{i} R\right)}{j_{l}\left(k_{i} R\right)}-\frac{\eta_{i} R}{m_{i 2}} \frac{k_{l+1}\left(\eta_{i} R\right)}{k_{l}\left(\eta_{i} R\right)}-l \frac{m_{i 2}-m_{i 1}}{m_{i 2} m_{i 1}}=0$

The energy levels of electron and hole confined $s$ states (i.e., with the quantum number $l=0$ ) in $\mathrm{Si}_{-} \mathrm{SiO}_{2}$ QD of $1.5 \mathrm{~nm}$ in diameter are shown in Fig. 1 by dotted lines.

By determining the coefficients $C_{i, k}^{\alpha}$ and energies $\tilde{E}_{i, \alpha}$ as a result of expansion (15) substitution into Eq. (14) and fulfilment of corresponding integrations, we completely solve the problem of finding the basic set of single-particle states $\Psi_{\alpha}^{i}$ in semiconductor QD inside this dielectric matrix. These states will be used below for a description of exciton and trion states in spherical QDs in the presence of the image forces field. The energies $E_{i}$ $(I=e, h)$ in the right-hand part of the expression (1) are expressed therewith as

$E_{i}=U_{s}(0)+\tilde{E}_{i, 0}$.
The Hamiltonian of a two-particle system, which determines the energies of exciton transitions in such QD, has the following form:

$$
\begin{aligned}
& \hat{H}_{X}=E_{g}+2 U_{s}(0)-\frac{\hbar^{2}}{2 m_{e}\left(r_{e}\right)} \Delta_{e}-\frac{\hbar^{2}}{2 m_{h}\left(r_{h}\right)} \Delta_{h}+ \\
& +\tilde{U}_{s}\left(r_{e}\right)+\tilde{U}_{s}\left(r_{h}\right)+\tilde{U}_{c}\left(r_{e}\right)+\tilde{U}_{v}\left(r_{h}\right)+U_{e h}\left(\mathbf{r}_{e}, \mathbf{r}_{h}\right)= \\
& =E_{g}+2 U_{s}(0)+\hat{H}_{e}+\hat{H}_{h}+U_{e h}\left(\mathbf{r}_{e}, \mathbf{r}_{h}\right),
\end{aligned}
$$

where the term $U_{e h}\left(\mathbf{r}_{e}, \mathbf{r}_{h}\right)=-e^{2} G\left(\mathbf{r}_{e}, \mathbf{r}_{h}\right)$ allows for both direct Coulomb interaction between an electron and a hole and indirect (i.e., interaction of an electron with the image of a hole and a hole with the image of an electron).

We will seek the wave function of the ground state of exciton in the form of a product of the "exciton" singleparticle electron and hole wave functions $\Psi_{e}^{X}\left(\mathbf{r}_{e}\right)$ and $\Psi_{h}^{X}\left(\mathbf{r}_{h}\right)$

$\Phi_{X}\left(\mathbf{r}_{e}, \mathbf{r}_{h}\right)=\Psi_{e}^{X}\left(\mathbf{r}_{e}\right) \Psi_{h}^{X}\left(\mathbf{r}_{h}\right)$,

where $\Psi_{e}^{X}\left(\mathbf{r}_{e}\right)$ and $\Psi_{h}^{X}\left(\mathbf{r}_{h}\right)$, in turn, can be conveniently presented in the form of an expansion in basal single-particle wave functions $\Psi_{\alpha}^{i}(15)$ :

$\Psi_{i}^{X}\left(\mathbf{r}_{i}\right)=\sum_{\alpha} B_{i}^{\alpha} \Psi_{\alpha}^{i}\left(\mathbf{r}_{i}\right)$

In this case, the Schroedinger equation for the ground state of exciton in a QD can be written in the form of a coupled equations for the electron and hole wave functions $\Psi_{i}^{X}$ :

$\left(\hat{H}_{e}+\frac{1}{2} \int U_{e h}\left(\mathbf{r}_{e}, \mathbf{r}_{h}\right)\left|\Psi_{h}^{X}\left(\mathbf{r}_{h}\right)\right|^{2} d \mathbf{r}_{h}\right) \Psi_{e}^{X}\left(\mathbf{r}_{e}\right)=$ $=\tilde{E}_{e}^{X} \Psi_{e}^{X}\left(\mathbf{r}_{e}\right)$

and

$\left(\hat{H}_{h}+\frac{1}{2} \int U_{e h}\left(\mathbf{r}_{e}, \mathbf{r}_{h}\right)\left|\Psi_{e}^{X}\left(\mathbf{r}_{e}\right)\right|^{2} d \mathbf{r}_{e}\right) \Psi_{h}^{X}\left(\mathbf{r}_{e}\right)=$ $=\tilde{E}_{h}^{X} \Psi_{h}^{X}\left(\mathbf{r}_{\boldsymbol{e}}\right)$

The system of equations (22) is solved selfconsistently in the way analogous to Hartree-Fock technique. As a first approximation by integrating in Eqs (22), the wave functions (15) of electron and hole ground states are taken: $\Psi_{i}^{X, 0}\left(\mathbf{r}_{i}\right)=\Psi_{\alpha=0}^{i}\left(\mathbf{r}_{i}\right)$. Further, in the basis of the functions $\Psi_{\alpha}^{i}\left(\mathbf{r}_{i}\right)$ (i.e., using the expansion (21)), we write Eq. (22) in a matrix form, diagonalize the matrix obtained, and find new wave functions of an electron and a hole $\Psi_{i, 0}^{X, 1}\left(\mathbf{r}_{i}\right)$ of the type (21). They correspond to the lowest one-particle 
energy states $\tilde{E}_{i, 0}^{X, 1}$ at a first iteration (the upper index 1 means that these functions and energies are obtained as a result of a first iteration, the lower index 0 that they correspond to the smallest eigenvalue of the diagonalized matrix, i.e., to the ground state). With these new wave-functions, we repeat integrations in (22), build new matrices and so on. The iterative procedure is continued until the relation of the energy increment obtained in the course of the last iteration to the total value of the energy obtained up to this moment becomes smaller than a preset accuracy. As a rule, to have the accuracy of the order of $10^{-6}$, three or four iterations are enough. As a result, we obtain "exciton" single-particle energies $\tilde{E}_{e}^{X}$ and $\tilde{E}_{h}^{X}$ in the exciton ground state. The energy of the system transition from a non-excited state into the exciton ground state (or vice versa) is expressed in this case as $E_{X}=E_{g}+2 U_{s}(0)+\tilde{E}_{e}^{X}+\tilde{E}_{h}^{X}$ (or $E_{X}=E_{g}+E_{e}^{X}+E_{h}^{X}$, if similarly to (18) we set $\left.E_{i}^{X}=U_{s}(0)+\tilde{E}_{i}^{X}\right)$. By calculating the "exciton" singleparticle energies $E_{i}^{X}$ and real single-particle energies $E_{i}$ (18) as well as comparing the above expression for the exciton transition energy with the expression (1), we are able thus to determine the binding energy $S_{X}$ of an exciton in a QD with finite band-off-sets and a polarized heterointerface:

$S_{X}=E_{e}+E_{h}-\left(E_{e}^{X}+E_{h}^{X}\right)$.

Fig. 2 shows calculated dependences of the exciton transition energy (a) and exciton binding energy (b) on the QD diameter for the cases of silicon QDs and those of some $\mathrm{A}^{\mathrm{II}} \mathrm{B}^{\mathrm{VI}}$ materials located in the $\mathrm{SiO}_{2}$ matrix. Within the considered range of QD diameters, the behaviour of the curves in Fig. 2a can be described as $E_{X}=E_{g}+A d^{-n}$, where $d$ is the QD diameter, and the parameter $n$ takes the following values: $1.461(\mathrm{Si}), 1.582$ (CdS), 1.271 (CdSe), and 1.273 (CdTe). Noteworthy is that in the infinite barrier height approximation these dependences should behave practically like $d^{-2}$. As seen from Fig. 2b, due to the effects of quantum confinement and dielectric enhancement, the binding energy of excitons in QD of small size $(\sim 1-2 \mathrm{~nm})$ can reach very large values (from several tenths to several electronvolts). A small difference between the exciton binding energies in $\mathrm{Si}, \mathrm{CdSe}$ and CdTe QDs (in Fig. 2b corresponding curves are shown for simplicity by one solid line) is explained by the fact that dielectric constants of these materials are close to each other $(\varepsilon$ $\sim 10-12$ ). The curve for the CdS QD in Fig. 2b (dashed line) lie much lower because of the smaller value of the dielectric constant in $\mathrm{CdS}$ material $(\varepsilon \sim 5.4)$.
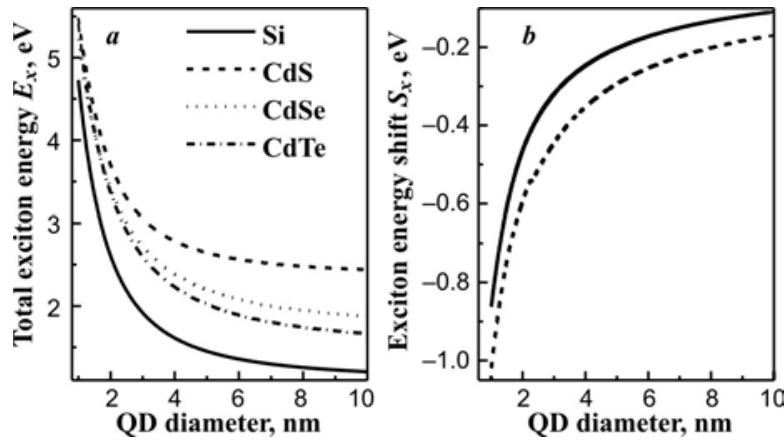

Fig. 2. Energies of the ground exciton transition in semiconductor QDs located in $\mathrm{SiO}_{2}$ matrix as functions of QD diameter (a). Exciton energy shifts in the exciton ground state (exciton binding energies taken with the negative sign) in semiconductor QDs located in $\mathrm{SiO}_{2}$ matrix as functions of QD diameter (b). Solid curve corresponds to the cases of $\mathrm{Si}, \mathrm{CdSe}$ and CdTe QDs, dashed one to CdS QD.

The described above technique may be also generalized to describe the trion states. As an example, let us consider the ground state of a negative trion in QD. Since in this case the electron subsystem should be a spin singlet, the wave function of a negative trion in a QD can be found in the form of a symmetrized product of "trion" single-particle functions $\Psi_{i}^{X^{-}}$:

$$
\begin{aligned}
& \Phi_{X^{-}}\left(\mathbf{r}_{1}, \mathbf{r}_{2}, \mathbf{r}_{h}\right)=\frac{1}{\sqrt{2}}\left[\Psi_{e, \alpha}^{X^{-}}\left(\mathbf{r}_{1}\right) \Psi_{e, \beta}^{X^{-}}\left(\mathbf{r}_{2}\right)+\right. \\
& \left.+\Psi_{e, \alpha}^{X^{-}}\left(\mathbf{r}_{2}\right) \Psi_{e, \beta}^{X^{-}}\left(\mathbf{r}_{1}\right)\right] \Psi_{h}^{X^{-}}\left(\mathbf{r}_{h}\right),
\end{aligned}
$$

where, analogously to the case of an exciton, "trion" single-particle functions will be presented in the form of an expansion in basic single-particle functions $\Psi_{\alpha}^{i}\left(\mathbf{r}_{i}\right)$ (15):

$$
\begin{aligned}
& \Psi_{e, \gamma}^{X^{-}}\left(\mathbf{r}_{i}\right)=\chi(\gamma) \sum_{\lambda} A_{e}^{\lambda} \Psi_{\lambda}^{e}\left(\mathbf{r}_{i}\right), \\
& \Psi_{h}^{X^{-}}\left(\mathbf{r}_{h}\right)=\sum_{\lambda} A_{h}^{\lambda} \Psi_{\lambda}^{h}\left(\mathbf{r}_{h}\right) .
\end{aligned} .
$$

In the expressions (24) and (25), the indices $\alpha, \beta$ and $\gamma$ are the spin variables taking the values $+1 / 2$ and $-1 / 2$, $\chi(\gamma)$ is an electron spin function. Although the spin coordinate will not be taken into account further in an explicit form, for a more accurate and rapid iteration it is worth distinguishing functions of electrons with different spins as it is made in the spin-unrestricted Hartree-Fock method [31, 32]. For such a form of the wave function $\Phi_{X^{-}}\left(\mathbf{r}_{1}, \mathbf{r}_{2}, \mathbf{r}_{h}\right)$, the Schroedinger equations for a negative trion in a QD may be written in the form of the following system of equations: 


$$
\begin{aligned}
& {\left[\hat{H}_{e}\left(\mathbf{r}_{1}\right)+\frac{1}{2} \int U_{e h}\left(\mathbf{r}_{1}, \mathbf{r}_{h}\right)\left|\Psi_{h}^{X^{-}}\left(\mathbf{r}_{h}\right)\right|^{2} d \mathbf{r}_{h}+\right.} \\
& \left.+\frac{1}{2} \int U_{e e}\left(\mathbf{r}_{1}, \mathbf{r}_{2}\right)\left|\Psi_{e, \beta}^{X^{-}}\left(\mathbf{r}_{2}\right)\right|^{2} d \mathbf{r}_{2}\right] \times \\
& \times \Psi_{e, \alpha}^{X^{-}}\left(\mathbf{r}_{1}\right)=\tilde{E}_{e, \alpha}^{X^{-}} \Psi_{e, \alpha}^{X^{-}}\left(\mathbf{r}_{1}\right), \\
& {\left[\hat{H}_{e}\left(\boldsymbol{r}_{2}\right)+\frac{1}{2} \int U_{e h}\left(\boldsymbol{r}_{2}, \boldsymbol{r}_{h}\right)\left|\Psi_{h}^{X^{-}}\left(\boldsymbol{r}_{h}\right)\right|^{2} d \mathbf{r}_{h}+\right.} \\
& \left.+\frac{1}{2} \int U_{e e}\left(\mathbf{r}_{1}, \mathbf{r}_{2}\right)\left|\Psi_{e, \alpha}^{X^{-}}\left(\mathbf{r}_{1}\right)\right|^{2} d \mathbf{r}_{1}\right] \times \\
& \times \Psi_{e, \beta}^{X^{-}}\left(\mathbf{r}_{2}\right)=\tilde{E}_{e, \beta}^{X^{-}} \Psi_{e, \beta}^{X^{-}}\left(\mathbf{r}_{2}\right), \\
& {\left[\hat{H}_{h}\left(\mathbf{r}_{h}\right)+\frac{1}{2} \int U_{e h}\left(\boldsymbol{r}_{1}, \boldsymbol{r}_{h}\right)\left|\Psi_{e, \alpha}^{X^{-}}\left(\boldsymbol{r}_{1}\right)\right|^{2} d \mathbf{r}_{1}+\right.} \\
& \left.+\frac{1}{2} \int U_{e h}\left(\mathbf{r}_{2}, \mathbf{r}_{h}\right)\left|\Psi_{e, \beta}^{X^{-}}\left(\mathbf{r}_{2}\right)\right|^{2} d \mathbf{r}_{2}\right] \times \\
& \times \Psi_{h}^{X^{-}}\left(\mathbf{r}_{h}\right)=\tilde{E}_{h}^{X^{-}} \Psi_{h}^{X^{-}}\left(\mathbf{r}_{h}\right),
\end{aligned}
$$

where $U_{e e}\left(\mathbf{r}_{1}, \mathbf{r}_{2}\right)=e^{2} G\left(\mathbf{r}_{1}, \mathbf{r}_{2}\right)$ includes both direct and indirect Coulomb interaction between two electrons in a trion. The single-particle wave functions $\Psi_{e, \gamma}^{X^{-}}$and $\Psi_{h}^{X^{-}}$in the ground state of trion and the corresponding energies $\tilde{E}_{i}^{X^{-}}$can be determined using the iteration procedure analogous to that described in the case of an exciton. The energy of the system transition from a non-excited state, when only one charge carrier is present in the QD (electron in the case of a negative trion) into the ground state of trion (or vice versa) is expressed as $E_{X^{-}}=E_{g}+2 U_{s}(0)+\left(2 \tilde{E}_{e}^{X^{-}}-\tilde{E}_{e}\right)+\tilde{E}_{h}^{X^{-}}$ (or $E_{X^{-}}=E_{g}+\left(2 E_{e}^{X^{-}}-E_{e}\right)+E_{h}^{X^{-}}$, if we introduce a designation $\left.E_{i}^{X^{-}}=U_{s}(0)+\tilde{E}_{i}^{X^{-}}\right)$. Then, it follows from the definition (2) that the energy shift of such a trion transition with respect to the exciton transition energy is expressed by the formula

$$
S_{X^{-}}=E_{e}+E_{e}^{X}+E_{h}^{X}-\left(2 E_{e}^{X^{-}}+E_{h}^{X^{-}}\right) .
$$

Similar expressions can be also obtained for the case of a positively charged trion.

Fig. 3 shows the dependence on the silicon QD diameter of the trion transition energy shift $-S_{X^{-(+)}}$ with respect to the exciton transition energy. As seen from this figure, if the effect of the heterointerface polarization is not taken into account, then the trion states in QD are more energetically favourable than that of an exciton, and with the QD size reducing their

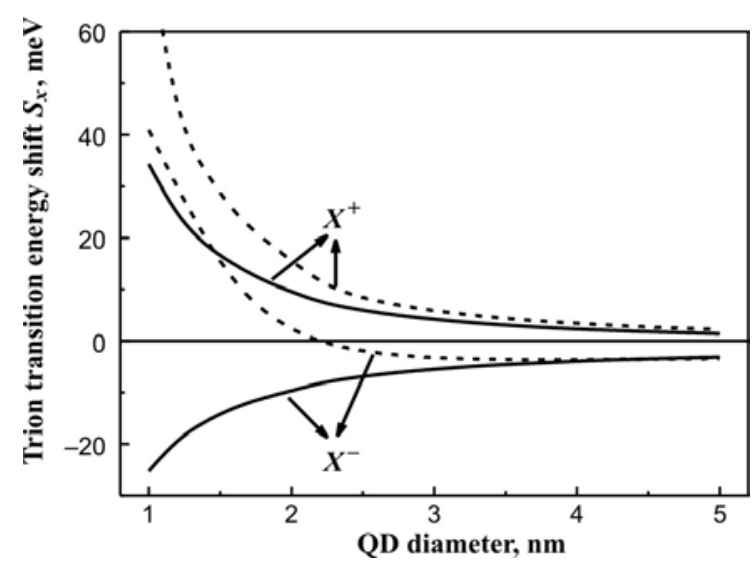

Fig. 3. Trion energy shifts in the trion ground state (trion binding energy taken with the negative sign) with respect to the energy of the state formed by non-interacting exciton and charge carrier in $\mathrm{Si}_{-} \mathrm{SiO}_{2} \mathrm{QD}$ as a function of the QD diameter. Solid curves correspond to the case when the effect of interface polarization is not taken into account, while dashed ones are obtained with such an account.

binding energy with respect to the exciton state increases and can reach the values of $10-20 \mathrm{meV}$ in QD of a very small size $(\sim 1-2 \mathrm{~nm})$. These values by factors of tens exceed trion binding energy in the case of bulk silicon [2]. The effect of the heterointerface polarization in $\mathrm{Si}$ $\mathrm{SiO}_{2}$ QDs considerably decreases the binding energy of trion states with respect to the exciton state in a QD of several nanometers in size. When the QD size decreases, the trion transition energy becomes even greater than that of the exciton, i.e., the trion state in principle is less energetically favourable in a small $\mathrm{Si}_{-} \mathrm{SiO}_{2}$ QD than the state in the form of a separate exciton and a charge carrier (e.g., those separated by tunneling into adjacent QDs).

\section{Conclusion}

Thus, in the present work by using the Hartree-Fock technique we have calculated the energy of the ground radiative exciton and trion transitions in semiconductor QDs located in a dielectric matrix. It has been shown that the trion transition energy can be either less or greater than the exciton transition energy depending on the physical parameters of the system under consideration.

For example, in the case of a $\mathrm{Si}_{-} \mathrm{SiO}_{2} \mathrm{QD}$ the barrier for holes due to the valence band-off-set is higher than the barrier for electrons $(\sim 4.6$ and $\sim 3.2 \mathrm{eV}$, respectively). Besides, the effective mass of a heavy hole in silicon $\left(\sim 0.5 m_{0}\right)$ is greater than that of an electron $\left(\sim 0.25 m_{0}\right)$. This makes hole states to be more localized in a QD than those of electrons. Therefore, the more effective repulsion of two holes in the case of a positively charged trion results in greater energies of the trion transitions in $\mathrm{Si}_{-} \mathrm{SiO}_{2}$ QDs than in the case of a negatively charged trion. 
It has been shown that if we do not take into account the image forces field that arises due to a substantial mismatch in the low-frequency dielectric constants of silicon $\left(\varepsilon_{1} \sim 11.7\right)$ and silicon dioxide $\left(\varepsilon_{2} \sim 2.1\right)$, the energy of the radiative transition in the case of a negatively charged trion is less than that of the exciton transition. This means that the effective attractive electron-hole interaction in such a trion exceeds the effective repulsive electron-electron interaction, and with the QD size decreasing, the trion state is becoming energetically ever more favourable as compared to the state of non-interacting exciton and electron. As far as a positively charged trion concerned, in the considered QD size range the trion transition energy is greater than that of the exciton, and with size reduction, the positive difference between the trion and exciton transition energies increases. Taking into account the polarization of the heterointerfaces causes a shift of the trion transition energies towards the short-wave region. Moreover, for small QD sizes $(<2 \mathrm{~nm})$, the transition energies of negatively charged trions become greater than the exciton transition energies (like to the case of positively charged trions within the whole range of considered QD sizes).

\section{Acknowledgements}

This work was supported in part by State Fundamental Researches Foundation of Ukraine, Russian-Ukrainian Program "Nanophysics and Nanoelectronics" and Program "Nanostructured Systems, Nanomaterials and Nanotechnologies" NAS of Ukraine.

\section{References}

1. M.A. Lampert, Mobile and immobile effectivemass-particle complexes in nonmetallic solids // Phys. Rev. Lett. 1 (12), p. 450-453 (1958).

2. T. Kawabata, K. Muro, S. Narita, Observation of cyclotron resonance absorptions due to excitonic ion and excitonic molecule ion in silicon // Solid State Communs 23 (4), p. $267-720$ (1977).

3. G.A. Thomas and T.M. Rice, Trions, molecules and excitons above the Mott density in Ge // Solid State Communs 23 (6), p. 359-363 (1977).

4. R.L. Greene, K.K. Bajaj, Binding energies of Wannier excitons in $\mathrm{GaAs} / \mathrm{Ga}_{1-\mathrm{x}} \mathrm{Al}_{\mathrm{x}} \mathrm{As}$ quantum well structures // Solid State Communs 45 (9), p. 831-835 (1983).

5. B. Gerlach, J. Wusthoff, M.O. Dzero, M.A. Smondyrev, Exciton binding energy in a quantum well // Phys. Rev. B58 (16), p. 10568-10577 (1998).

6. F. Rossi, G. Goldoni, O. Mauritz, E. Molinari, Theory of excitonic confinement in semiconductor quantum wires // J. Physics: Condens. Matt. 11 (31), p. 5969-5988 (1999).

7. E. Cho, M.A. Green, J. Xia, R. Corkish, P. Reece, M. Gal, Clear quantum-confined luminescence from crystalline Silicon $/ \mathrm{SiO}_{2}$ single quantum wells // Appl. Phys. Lett. 84 (13), p. 2286-2288 (2004).

8. M.G. Lisachenko, E.A. Konstantinova, P.K. Kashkarov, V.Yu. Timoshenko, Dielectric effect in silicon quantum wires // Phys. status solidi (a) 182 (1), p. 297-299 (2000).

9. Yu.V. Kryuchenko, A.V. Sachenko, Quantum efficiency of exciton luminescence in lowdimensional structures with indirect energy gap // Physica E 14, p. 299-312 (2002).

10. G. Finkelstein, H. Shtrikman, I. Bar-Joseph, Negatively and positively charged excitons in GaAs/ $/ \mathrm{Al}_{\mathrm{x}} \mathrm{Ga}_{1-\mathrm{x}} \mathrm{As}$ quantum wells // Phys. Rev. B53 (4), p. R1709-R1712 (1996).

11. A. Esser, E. Runge, R. Zimmerman, W. Langbein, Electron and hole trions in wide GaAs quantum wells // Phys. status solidi (b) 221 (1), p. 281-286 (2000).

12. A.V. Filinov, C. Riva, F.M. Peeters, Yu.E. Lozovik, M. Bonitz, Influence of well-width fluctuations on the binding energy of excitons, charged excitons, and biexcitons in GaAs-based quantum wells // Phys. Rev. B70, p. 035323-1-035323-13 (2004).

13. J.J. Palacios, D. Yoshioka, A.H. MacDonald, Longlived charged multiple-exciton complexes in strong magnetic fields // Phys. Rev. B54 (4), p. R2296R2299 (1996).

14. P. Kossacki, J. Cibert, D. Ferrand, Y. Merle d'Aubigné, A. Arnoult, A. Wasiela, S. Tatarenko, J.A. Gaj, Neutral and positively charged excitons: A magneto-optical study of a p-doped $\mathrm{Cd}_{1-\mathrm{x}} \mathrm{Mn}_{\mathrm{x}} \mathrm{Te}$ quantum well // Phys. Rev. B60 (23), p.1601816026 (1999).

15. A. Esser, E. Runge, R. Zimmermann, Photoluminiscence and radiative lifetime of trions in GaAs quantum wells // Phys. Rev. B62 (12), p. 8232-8239 (2000).

16. Israel Bar-Joseph, Trions in GaAs quantum wells // Semicond. Sci. Technol. 20, R29-R39 (2005).

17. W. Ossau, D.R. Yakovlev, C.Y. Hu, V.P. Kochereshko, G. V. Astakhov, R.A. Suris, P.C.M. Christianen, J.C. Maan, Exciton-electron interaction in quantum wells with a two dimensional electron gas of low density // Phys. Solid State 44, p. 751-756 (2002).

18. A. Esser, R. Zimmerman R, E. Runge, Theory of trion spectra in semiconductor nanostructures // Phys. status solidi (b) 227, p. 317-330 (2001).

19. R.A. Sergeev, R.A. Suris, The $X^{+}$trion in a system with spatial separation of the charge carriers // Semiconductors 37 (10), p. 1205-1210 (2003).

20. B. Stébé and A. Ainane, Ground state energy and optical absorption of excitonic trions in two dimensional semiconductors // Superlattices Microstruct. 5 (4), p. 545-548 (1989).

21. K. Kheng, R.T. Cox, Merle Y. d'Aubigné, Franck Bassani, K. Saminadayar, and S. Tatarenko, Observation of negatively charged excitons $\mathrm{X}^{-}$in 
semiconductor quantum wells // Phys. Rev. Lett. 71 (11), p. 1752-1755 (1993).

22. R.J. Warburton, C. Schäflein, D. Haft, F. Bickel, A. Lorke, K. Karrai, J.M. Garcia, W. Schoenfeld, P.M. Petroff, Optical emission from a chargetunable quantum ring // Nature (London) 405, p. 926-929 (2000).

23. A.S. Bracker, E.A. Stinaff, D. Gammon, M.E. Ware, J.G. Tischler, D. Park, D. Gershoni, A.V. Filinov, M. Bonitz, F. Peeters, C. Riva, Binding energies of positive and negative trions: From quantum wells to quantum dots // Phys. Rev. B 72 (3), p.035332-1035332-6 (2005).

24. L.V. Keldysh, Coulomb interaction in thin semiconductor and semimetal films // JETP Lett. 29, p. 658-661 (1979).

25. V.S. Babichenko, L.V. Keldysh, A.P. Silin, Coulomb interaction in thin semiconductor and semimetal wires // Soviet Phys.- Solid State 22, p. 723-729 (1980).

26. A. Orlandi, R. Rontani, G. Goldoni, F. Manghi, E. Molinari, Single-electron charging in quantum dots whis large dielectric mismach // Phys. Rev. B63, p. 045310-045316 (2001).
27. V.A. Fonoberov and E.P. Pokatilov, A.A. Balandin, Exciton states and optical transitions in colloidal CdS quantum dots: Shape and dielectric mismatch effects // Phys. Rev. B66, p. 085310-1 - 085310-12 (2002).

28. D.V. Korbutyak, Yu.V. Kryuchenko, A.V. Sachenko, I.M. Kupchak, Characteristics of confined excitons in silicon quantum wires // Semiconductor Physics, Quantum Electronics \& Optoelectronics 6 (2), p. 172-182 (2003).

29. J.D. Jackson, Classical electrodynamics. John Wiley \& Sons Inc., New York - London (1962).

30. B.I Boichuk, P.Yu. Kubay, I.V. Bilynskii, Influence of the image potential on the energy spectrum of electron in complex spherical microcrystal $\mathrm{CdS} / \beta$ $\mathrm{HgS} / \mathrm{H}_{2} \mathrm{O} / /$ J. Phys. Studies 3(2), p. 187-191 (1999) (in Ukrainian).

31. J.R. Trail, M.D. Towler, and R.J. Needs, Unrestricted Hartree-Fock theory of Wigner crystals // Phys. Rev. B68, p. 045107-045112 (2003).

32. C. Yannouleas and U. Landman, Spontaneous symmetry breaking in single and molecular quantum dots // Phys. Rev. Lett. 82 (26), p. 5325 5328 (1999). 